AperTO - Archivio Istituzionale Open Access dell'Università di Torino

\title{
On a Modeling Approach to Analyze Resilience of a Smart Grid Infrastructure
}

\section{This is the author's manuscript}

Original Citation:

Availability:

This version is available http://hdl.handle.net/2318/150057

since

Publisher:

IEEE

Terms of use:

Open Access

Anyone can freely access the full text of works made available as "Open Access". Works made available under a Creative Commons license can be used according to the terms and conditions of said license. Use of all other works requires consent of the right holder (author or publisher) if not exempted from copyright protection by the applicable law. 


\section{(6) \\ UNIVERSITÀ DEGLI STUDI DI TORINO}

This is an author version of the contribution published on:

Questa è la versione dell'autore dell'opera:

Dependable Computing Conference (EDCC), 2014 Tenth European, 2014, 10.1109/EDCC.2014.34

The definitive version is available at:

La versione definitiva è disponibile alla URL:

http://ieeexplore.ieee.org/xpl/freeabs_all.jsp?arnumber=6821102\&abstractAccess= no\&userType=inst 


\title{
On a Modeling Approach to Analyze Resilience of a Smart Grid Infrastructure
}

\author{
Silvano Chiaradonna, Felicita Di Giandomenico and Nadir Murru \\ ISTI-CNR, Pisa, Italy \\ Email: silvano.chiaradonna@isti.cnr.it, felicitadigiandomenico@isti.cnr.it, nadir.murru@isti.cnr.it
}

\begin{abstract}
The evolution of electrical grids, both in terms of enhanced ICT functionalities to improve efficiency, reliability and economics, as well as the increasing penetration of renewable redistributed energy resources to favor sustainability of the production and distribution of electricity, results in a more sophisticated electrical infrastructure which poses new challenges from several perspectives, including resilience and quality of service analysis. In addition, the presence of interdependencies, which more and more characterize critical infrastructures (including the power sector), exacerbates the need for advanced analysis approaches, to be possibly employed since the early phases of the system design, to identify vulnerabilities and appropriate countermeasures. In this paper, we outline an approach to model and analyze smart grids and discuss the major challenges to be addressed in stochastic model-based analysis to account for the peculiarities of the involved system elements. Representation of dynamic and flexible behavior of generators and loads, as well as representation of the complex ICT control functions required to preserve and/or re-establish electrical equilibrium in presence of changes (both nominal ones, such as variable production by a photovoltaic energy source, and failures/disruptions both at electrical and ICT level) need to be faced to assess suitable indicators of the resilience and quality of service of the smart grid.
\end{abstract}

\section{INTRODUCTION AND RELATED WORK}

Smart grids aim at evolving the traditional electrical grid system by introducing sophisticated ICT control functionalities to improve efficiency, reliability and economics, as well as the increasing penetration of renewable distributed energy resources to favor sustainability of the production and distribution of electricity. This results in a more sophisticated electrical infrastructure which poses new challenges from several perspectives, including resilience and quality of service analysis. In addition, the presence of interdependencies, which more and more characterize critical infrastructures (including the power sector), exacerbates the need for advanced analysis approaches, to be possibly employed since the early phases of the system design, able to represent and master an integrated vision of the entire system, seen as a system of systems with intricate relationships among them.

Stochastic model-based analysis [1] is widely applied as an early validation technique in a variety of studies, including those focusing on resilience and, in general, quality of services properties. Its reduced cost, compared to measurement-based methods, makes it an attractive alternative to analyze a system towards identification of weaknesses and vulnerabilities, so that means to enhance system robustness can be identified and put in place. To account for interdependencies existing among the major infrastructures composing an electrical power system, the system model needs to include all the involved components, to trace the propagation of phenomena affecting individual parts. The resulting complexity needs to be managed from several perspectives, including resorting to an appropriate abstraction level of the involved structural and behavioural aspects, as well as promoting modular and compositional approaches to model development.

In this paper, we address the analysis of smart grids in terms of indicators representative of their resilience as perceived by final customers as well as distribution system operators. The results of this kind of analysis can be exploited to understand the dynamics of failures and potential system vulnerabilities, against which appropriate countermeasures need to be identified. The objective of the work is to build a general and composable modeling framework, populated by template building blocks which represent models of components/events, so as to be able to account for a variety of grid configurations and critical situations characterized by failure events, in presence of interdependencies. Fulfilling such objective requires significant effort, both in resources and time, and is part of the contribution planned by the ongoing European project SmartC2Net (https://intern.smartc2net.eu). The current developments tackle the work from a logical and systematic angle, by i) identifying the abstracted system architecture to be modeled, whose components are characterized by both a state and a behaviour; ii) introducing relevant analysis indicators; and iii) exploring modeling approaches able to trade between efficiency of the solution and accuracy in modeling a variety of smart grid configurations and sophisticated control functionalities. The challenges raised by the dynamic and flexible behaviour (as shown by renewable energy resources and by categories of loads at the medium and low voltage level), as well as by the crucial electrical production-consumption equilibrium in the smart distribution grid are discussed, with reference to their implications on the modeling and solution approach. These are fundamental steps towards a sound modeling and analysis framework for smart grids.

Previous studies have pursued similar objectives, but focusing on interdependencies at the level of the electric transmission grid, such as [2]-[5]. Although we get inspiration from previous experience with modeling the transmission segment, here we account for the volatility of the microgrid generation and newly appliances to control and manage distribution of electricity to medium and low voltage loads. With reference to the distribution grid, most of the proposals in the literature address the modeling of cyber attacks to reveal vulnerabilities, perform impact analysis and assess the cyber risk, e.g. [6]-[8]. Our framework targets a wider characterization of the fault model, 
including both accidental faults, affecting either the ICT control infrastructure or the electric grid, and cyber attacks to the ICT control, in addition to a special focus on the reciprocal dependencies originating failure cascading effects. Simulation studies also offer an alternative approach able to account for both the electrical grid and the smart ICT control for analyzing the dynamics and mutual impacts of both domains, such as [9], where however the focus is on the evaluation of real-time performance indicators, while our objective is to assess a wider class of resilience and QoS indicators, explicitly accounting for failures and their propagation.

The rest of the paper is organized as follows. Section II presents the main logical components of the smart grid to be considered in the modeling framework, the characterization of their state and their relationships. Section III describes the fault model assumed, as well as the interdependencies due to the interconnections among the electrical distribution grid and its control subsystem. A set of resilience-related metrics of interest for the analysis is included in Section IV, while discussion and identification of the approach to build the modeling framework, with preliminary exemplifications using the SAN formalism are carried on in Section V. Finally, conclusions are drawn in Section VI.

\section{Logical Structure of the SMart Grid System}

In this section, we describe the main logical components of the smart grid (SG) to be considered in the modeling framework and their relationships. The proposed logical structure of the SG has been derived from the description of the architecture [10] and of the use cases [11]. The focus is on SG at level of electrical distribution systems.

The considered SG is logically structured in two cooperating parts, as shown in Figure 1a: the Electric Infrastructure (EI) and the Monitoring and Control System (MCS) based on the Information and Communications Technologies (ICT).

\section{A. The Electrical Distribution Infrastructure}

The EI represents the electrical infrastructure, which is responsible for generating electric power at medium and low voltage, and for transporting towards the final users the electric power received from the transmission system and generated at medium and low voltage. The EI is structured in two segments: (i) MV-EI: the medium voltage electrical distribution infrastructure (with an operating voltage of $10 \mathrm{kV}$ $60 \mathrm{kV}$ ) physically connected to the high voltage (HV-EI) and low voltage electrical infrastructures. (ii) LV-EI: the low voltage electrical distribution infrastructure (with an operating voltage of 220V-380V), physically connected to MV-EI. MV-EI and LV-EI can be logically structured in:

- Power stations (generators), i.e., sources of energy connected at substations to supply energy to the distribution network.

- Load stations (loads), i.e., equipment connected at substations to extract energy from the distribution network.

- Substations, i.e., an assembly of elecrical equipment that allows the routing and control of electricity across the network [12], e.g., substations control the voltage and direction of electricity; they generally have switching, protection and control equipment, and transformers; substations themselves do not usually have generators, although a power plant may have a substation nearby; other elecrical devices such as capacitors and voltage regulators may also be located at a substation.

- A combination of the above items (these components will be referred as substations), e.g., a logical component of MV-EI can be composed by a generator, a load and a substation where a capacitor bank is located.

- Power lines, i.e., the electrical circuits (e.g., overhead lines and cables), connecting stations and substations.

From a topological point of view, the infrastructures MV-EI and LV-EI can be considered like networks, or graphs, as shown in the example of diagram of Figure $1 \mathrm{~b}$.

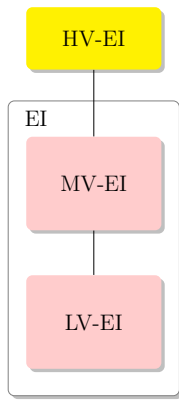

(a)

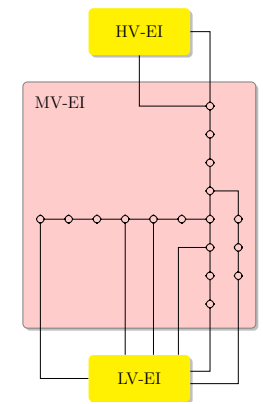

(b)

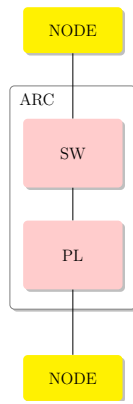

(c)
Fig. 1. Logical scheme of EI (a), example of logical scheme of MV-EI with radial topology (b) and logical scheme of an arc of MV-EI and LV-EI (c).

The topology of the network is typically a radial graph, Figure (1b) or, for redundancy purposes, a partially/weakly meshed (e.g., loop/ring, selective or spot) graph. An arc (or branch) of the graph represents a power line with the associated switch and protection breakers, if any, while a node represents a power station, a load station or a substation (or a combination of them). Substations (nodes of the network) of MV-EI directly connected to the HV-EI through high-voltage power lines, are primary substations, i.e., substations that reduce the high voltage to a voltage suitable for MV-EI. Substations of LV-EI directly connected to the MV-EI through medium-voltage power lines, are secondary substations, i.e., substations that reduce the medium voltage to a voltage suitable for LV-EI. In MV-EI, high-voltage transmission lines feeding a primary substation are considered like power sources connected directly to the primary substation (on the primary bus of the transformer); low-voltage distribution lines feeding secondary substations are considered power loads connected directly to the secondary substation. In LV-EI, medium-voltage distribution lines feeding a secondary substation are considered like power sources connected directly to the secondary substation.

The logical structure of a generic arc is shown in Figure 1c, where a power line (PL) with the associated protection units, including breakers, and the associated switch (SW) are considered. Changing the state of a switch, it is possibile to reconfigure the distribution system, in order to modify the topology of the network.

For simplicity of representation, each generic node, representing a station or substation, can be structured like a busbar (bus) with the associated elecrical equipment. Figure 2 
shows the logical scheme adopted for a generic node of MVEI. Protection units (including breakers), which are physically

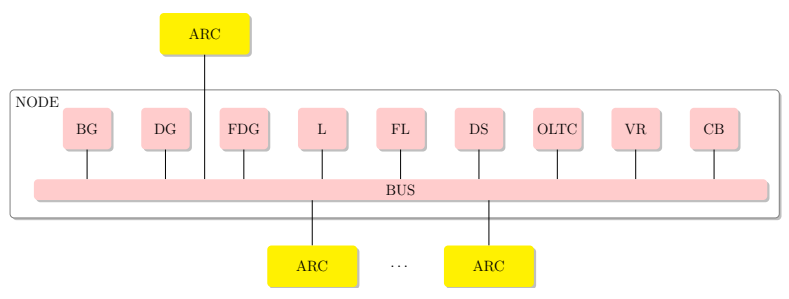

Fig. 2. Logical scheme of a node of MV-EI. In a real world instance of the node, only a subset of the components connected to BUS is considered.

part of a substation, are not explicitally represented in the considered logical scheme, but they are implicitely included in the logical components. The logical structure of a specific power station, load station or substation may be obtained considering a subset of the logical components associated to the generic node, depending on the real structure of the considered component.

At the level of abstraction considered, the logical structure of a generic node of MV-EI is based on the following components:

- Bulk generators (BG): classic no dispersed generators can produces elecrical energy in bulk quantity.

- Distributed generators (DG): volatile small-scale energy generating units, producing electricity from, for example, renewable energy sources (RES), i.e., wind, hidro, biomass, solar/photovoltaic, etc.

- Flexible distributed generators (FDG): generators that offer flexibility in the power profile, e.g., shifting in time, changing the energy amount or changing the tariff [13].

- Non flexible loads (L): classic loads for which a loss of power is a blackout, because the power demand cannot shift in time or change in the required energy amount.

- Flexible loads (FL): loads that offer flexibility in the power profile, e.g., shifting in time (i.e., shifting loads to less expensive time slots), changing the energy amount or changing the tariff; for flexible loads the loss of power is not a blackout if the power profile can shift in time; examples of flexible loads are: electrical charging stations (ECS) or charging spots (CS) (depending on the considered level of detail), enterprises, etc.

- Distributed storage units (DS): electrical devices that can be considered alternatively (flexible) generator or (flexible) load, depending on the state of the power system; they can be placed near to renewable energy systems to smooth their generation profile, or they can be directly operated by the distribution system operators (DSO) to enhance network performances, i.e., to help to supply peak power and to improve power quality (e.g., through voltage regulation) [14], [15]. "In general, energy storage systems use electricity during non-peak hours or from intermittent sources, and the stored energy is converted back to electricity for the loads during peak periods; the price difference between energy during peak and off-peak hours is the main motivation for installing DS units" [16],

- Transformers with On load tap changers (OLTC): transformers having voltage regulators at primary substations.

- Voltage regulators (VR).

- Capacitor banks (CB): voltage regulators based on the injection of reactive power.
Generation, energy storage and load systems that are connected to a power distribution system are defined as generic distributed energy resource (DER).

The logical structure of a generic node of LV-EI is based on the following components:

- DG, FDG, DS, L, FL (like electrical vehicle charging, that can be used by the local DSO to manage power quality control in the LV grid along with decentralized PV production as well as other loads, e.g. households [17]), Transformer (T) having off-load tap changers.

- Micro/mini generators (MG): volatile small-scale energy generating units, producing electricity at lower voltage levels, e.g., from wind, solar/photovoltaic, etc.; MG are under the customer domain and, in conventional systems, can not be remote controlled by an operator; in the following it is assumed that MG can be remote controlled by an operator.

Traditionally, OLTC, capacitor banks and remotely controlled switches are not available at LV-EI, therefore, only controlling load and active power injection, can voltage be kept within regulatory limits [18]. Consequently, OLTC, voltage regulators and capacitor banks are not considered in the logical scheme of the node, although, it can be extended to include them.

\section{B. The Monitoring and Control System based on the Informa- tion and Communications Technologies}

The MCS represents the system that monitors and controls the physical parameters of the electric infrastructure, and triggers appropriate reconfigurations when needed (e.g., in emergency situations). The main objectives of the MCS are:

- to balance production and consumption as locally as possible in order to avoid transmission losses,

- to increase transmission reliability, through ancillary services such as voltage/var support, switches reconfiguration, generator redispaching and load shedding.

Control and automation functions are no longer limited to control center and appear throughout the network. They are hierarchically organized, with control layers corresponding to the main voltage levels. Thus, MCS is hierarchically structured in three main logical components, shown in Figure 3:

- CMCS: grid central management and control systems, that, at the considered level of abstraction, include internal systems (DSO Operation and Enterprise Centers, Demand Management Control, Tariff Management, TSO) and external systems (Weather Forecast, Aggregator Controller, Distribution Market, Information Service and Charging Station and Routing Reservation).

- MV-MCS: medium voltage monitoring and control system, - LV-MCS: low voltage monitoring and control system.

All the control operations performed by MCS on EI are not represented in detail, but a simplified model is considered where only the effects on the distribution grid of the mitigation methods to cope with EI malfunctions, namely generation redispatch, load shedding, grid reconfigurations or voltage/var control are accounted for. Figure 3 depicts a possible detailed logical structure of MCS. The components CSYS, MVGC, 




Fig. 3. Detailed logical scheme of MCS.

LVGC and LC differ for the locality of their decisions and can exchange grid status information and control data over public or private networks (INTERNET, WAN and AN). The main functions of the component MVGC and LVGC are:

- to monitor their assigned area in order to diagnose faults in the electrical/control components,

- to choose the most suitable corrective actions to restore the functionality of the grid, in case of faults.

Since they are not directly connected to the controlled components, the corrective actions are put in operation through the pertinent logical controller (LC), i.e., an ICT-based component having the ability to monitor/control an electrical component. LC guarantee the correct operation of each controlled component (generators, loads, storage units, etc.) and reconfigure it in case of fault of some apparatus. At the level of abstraction considered, LC includes the data acquisition and control equipment (sensors and actuators) and also smart meter or Customer Energy Management System (CEMS), if needed.

At medium voltage, one MVGC is associated to each different primary substation. MVGC monitors and controls all the medium-voltage electrical components connected (directly or indirectly) to the primary substation, i.e., the components located along the feeders emanating from the primary substation, and that can be remotely controlled by an operator. At low voltage, one LVGC is associated to each different secondary substation. LVGC monitors and controls all the low-voltage electrical components connected to the secondary substation. At medium and low voltage, an LC is associated to each electrical component that can be directly controlled by an LC. Therefore, at each substation (corresponding to a node of the EI network) at medium and low voltage is associated: one logical component
$M V G C$, or one logical component $L V G C$ or one or more logical components $L C$.

Figure 4 shows all the electrical components, at low voltage, under the direct control of an operator. The logical component



Fig. 4. Detailed logical scheme of LV-MCS.

MVG represents the power source corresponding to a mediumvoltage distribution line feeding a secondary substation. MVG is not under the direct control of LVGC, that receives the set points for MVG from MVGC. It can be assumed that, LC can operate in: (i) isolated/independent/non-coordinated mode, (ii) coordinated/central mode, or (iii) both isolated and coordinated mode.

Isolated mode occurs when each device is controlled independently, without regard for the resulting consequences of actions taken by other control devices [19] (traditional aproach used for voltage/var control devices); i.e., the LC operation is not coordinated with the other LC operations and new set points for the component controlled by LC can be defined independently by the operation (i.e., set points) of the other LC. With the isolated mode, a new timely, though inconsistent or suboptimal, configuration for EI can be obtained without the intervention of LVGC and MVGC (and CSYS).

Coordinated mode occurs when the operations of all the LC that control the components located along the feeders emanating from the same primary or secondary substation, are coordinated [20], respectively, by the MVGC or LVGC (with the contribution of CSYS) associated to the substation. With the coordinated mode, a new consistent and (more) optimal, though less timely or delayed, configuration for EI can be only obtained with the intervention of LVGC or MVGC.

Both isolated and coordinated modes occur when a new (timely, though inconsistent or suboptimal) configuration for EI is first obtained without the intervention of LVGC and MVGC, and next, a new (optimal, though delayed) configuration for EI is obtained with the intervention of LVGC or MVGC.

No LC is associated to electrical components that are not under the direct control of an operator; the state of these electrical components (e.g., the value of voltage) can change as a result of a reconfiguration of the controlled electrical components. If an electrical component can be controlled only in isolated/non-coordinated mode, then the associated LC is not connected to MVGC or LVGC by a communication network. Finally, notice that, since different electrical components can be associated on the same node of the EI network, then also different LC can be associated to the same node of the communication network. 
MCS actions can be abstracted at one or two levels on the basis of the locality of the EI state considered by MCS to decide on proper reactions to disruptions: (i) single LC local level (isolated mode): only the state local to the affected EI component is considered by each involved LC, (ii) MVGC/ LVGC global level (coordinated mode): the state global to all the affected EI system under the control of MVGC/LVGC is considered. Each level of the MCS actions is characterized by:

- an activation condition, specifying the events that enable the MCS reaction: disruption/faults, intermittency of renewable sources, power demand variation, etc.,

- a reaction delay, representing the overall computation and application time needed by MCS to apply a reconfiguration,

- a reconfiguration strategy (RS), based on generation redispatch (varying the generated power), load shedding (varying the load demand), voltage/var control, load balancing, power loss reduction, line overload reduction, etc.

The reconfiguration strategy RS defines how the configuration of EI changes when MCI reacts to an event that has compromised the electrical equilibrium. For each level, a different reconfiguration function can be considered.

\section{State Definition}

The state of EI is an hybrid-state composed by a discrete part and a continuous part. To represent the discrete part the following entities have to be considered:

- an oriented graph $T_{G}$, representing the topology of EI (as shown for example in Figure 1b), i.e., nodes and arcs with the associated components (as shown for example in Figures 1c and 2) and the direction of the current flow on each power line,

- the quantities representing the possible discrete settings of the electrical control devices, like those used to VOLT/ VAR control (OLTC, capacitor banks, etc.), i.e., for example, the value on/off for each capacitor and the tap position for each OLTC (over the number of available tap positions) considered in EI,

- the discrete quantities representing the correct behavior or failed bahavior/outage (due to an accidental or intentional fault) of the electrical components.

To represent the continuous part of the EI state the following quantities have to be considered: (i) the physical quantities $V$, $\delta_{V}, I, P$ and $Q$ associated with the equipment that constitutes the electric infrastructure (generators, loads, busses, power lines, etc.), i.e., respectively, voltage, voltage phase angle, current flow, active and reactive power, (ii) other quantities representing the possible continue value settings of the electrical devices different by those described in the above item (if any). Each state of EI can be described as a function of the entities described above. The state of MCS can be considered discrete, in the sense that it is only composed by discrete values. These values have to represent mainly the correct behavior or failed beviour (due to an accidental or maliocious fault) of the electrical components. Possibile values are: working, failure, omission failure, repairing, etc.

\section{Characterization and Behavior of Electrical Components}

In this section, the parameters/quantities, the characterization and the behavior of the main electrical components, that are considered in the proposed modeling framework, are presented and discussed.

1) Buses: Voltage $V_{h}(t)$, phase angle of the voltage $\delta_{V, h}(t)$, active power $P_{h}(t)$ and reactive power $Q_{h}(t)$ are associated to the bus $h$ at time $t$. In order that voltage variation may not degrade the customer voltage supply quality, feeder voltage should be maintained within the permissible range [20], [21], i.e.:

$$
V_{h}(t)-V_{h}(t) \epsilon \leq V_{h}(t) \leq V_{h}(t)+V_{h}(t) \epsilon,
$$

with $0 \leq \epsilon \leq 1$ (usually, rated voltage $+/-10 \%$, corresponding to $\epsilon=0.1$ ). When the voltage profile of a component reaches the statutory upper or lower limit, then individual protection of the component will trip for overvoltage/undervoltage causing the voltage to drop. This can propagate through the network causing other components to trip aggravating even more this problem and further reducing system voltage. The values of $P_{h}(t), Q_{h}(t), V_{h}(t)$ and $\delta_{V}(t)$ can be influenced by various factors, these being the power injection and absorption at bus $h$ by generators and loads (or storage), the current through the lines connected to node $h$, the power injection and absorption at bus $h$ by voltage and reactive power control devices.

2) Power Lines: Maximum power flow $I_{l}^{\max }$ that a power line can carry whitout being overloaded (capacity), power flow $I_{l}(t)$, impedance $Z_{h}$ (representing all forms of opposition to power flow), voltage drop $\Delta V_{l}$ and line power loss $\Delta S_{l}$ are associated to the power line $l\left(P L_{l}\right)$ at time $t$. When, at time $t$, the power flow exceedes the capacity, i.e., if $I_{l}(t)>I_{l}^{\max }$, then the line is overloaded. The overload, if not removed through a reconfiguration of EI, can lead to the trip of the line (if the power line protections open the line breaker) or to the disruption of the line (if the breaker fails to open); in the case of permanent disruption the repair of the power line is required.

3) Non Flexible Loads: The quantities considered for a non-flexible load on the bus $i\left(L_{i}\right)$ at time $t$ are: constant or variable power demand $D_{i}(t)$; power demand forecast $D_{i}^{F}(t)$; tariff/price (cost for the customer or reward for the operator) of the supplied electrical energy $C_{i}^{D}(t)$; rated voltage $V_{i}^{D}$; actual power demand $P_{i}^{D}(t)$ (active power) that is met (the power is supplied by no flexible and flexible generators); actual voltage $V_{i}(t)$ on the load; reactive power $Q_{i}^{D}(t)$; and load power demand that is not met $U D_{i}(t)=D_{i}(t)-P_{i}^{D}(t)$, due to an outage occurred in EI or a fault in MCS (a measure of a cost or blackout for the final customer or user). The quantity $D_{i}^{F}(t)$, that can be considered a random variable as a function of the time $t$, i.e., a stochastic process, represents the load forecast system. The quantity $D_{i}(t)-D_{i}^{F}(t)$ represents a measure of demand forecast error for the load associated to node $i$.

4) Flexible Loads and Flexibility Patterns: The quantities considered for a flexible load on the node $i\left(F L_{i}\right)$ at time $t$ are the same as those for the non-flexible loads described above, except that the power demand $D(t)$ is flexible. The power flexibility of the power demand can be exploited in three dimensions: time, energy amount or tariff for the user or the cost for the operator. For flexible loads, the power demand 
that is not met $U D_{i}(t)$ could not be considered a blackout for the final customer, or it could represent a cost lesser than that corresponding to a blackout, depending on the flexibility pattern adopted for $D_{i}(t)$.

A flexibility pattern represents the fexibility of a load in terms of intervals of time for which $U D_{i}(t)$ is (or is not) a blackout, or in general a cost, for the customer/operator, based on the tariff/cost associated to each interval.

A first flexibility pattern is described by $F P_{i}^{D}(t)=$ $\left(D_{i}(t),\left(t_{1}, l_{1}\right),\left(t_{2}, l_{2}\right)\right)$, where:

- $D_{i}(t)$ is the (constant or variable) power demand required at time $\mathrm{t}$,

- $\left(t_{1}, l_{1}\right)$ and $\left(t_{2}, l_{2}\right)$ are the non-overlapping time intervals,

- in the first time interval $\left(t_{1}, t_{1}+l_{1}\right)$ the power demand that is met $P_{i}^{D}(t)$ could be also less than $D_{i}(t)$,

- in the second time interval $\left(t_{2}, t_{2}+l_{2}\right) P_{i}^{D}(t)$ must be equal to $D_{i}(t)$

Therefore, in $\left(t_{1}, l_{1}\right) U D_{i}(t)$ is not a cost/blackout for the final customer, on the contrary in $\left(t_{2}, l_{2}\right) U D_{i}(t)$ is a cost/blackout for the final customer.

A second flexibility pattern is described by $F P_{i}^{D}(t, w)=$ $\left(D_{i}(t), w,\left(t_{1}, l_{1}, w_{1}\right),\left(t_{2}, l_{2}, w_{2}\right), \ldots,\left(t_{m}, l_{m}, w_{m}\right)\right)$, where $m$ different tariffs $w_{1}, \ldots, w_{m}$, are considered for $m$ different non-overlapping and consecutive time intervals, respectively, $\left(t_{1}, l_{1}\right), \ldots,\left(t_{m}, l_{m}\right)$. The customer agrees on the tariff $w=w_{h}$. Then, in the time intervals where a tariff $w$ or lower than $w$ is valid, i.e., for each $\left(t_{j}, l_{j}\right)$, such that, $w_{j} \leq w$, the customer requests the satisfaction of the demand $D_{i}(t)$; thus, in case the demand is not satisfied, a cost/blackout is incurred. On the contrary, in the time intervals where a tariff greater than $w$ is valid, i.e., for each $j$, such that $w_{j}>w$, the actual power demand (active power) that is to met $P_{i}^{D}(t)$ could be also less than $D_{i}(t)$; in this case, the lack of satisfied demand is not perceived as a blackout/cost. Notice that, another period of time with the corresponding tariff can be included, by considering the period complementary to the first $m$ intervals, i.e., a tariff $w_{m+1}$ for each time $t$, such that $t<t_{1}$ or $t_{j}+l_{j}<t<t_{j+1}$, for $j=2, \ldots, m-1$, or $t>t_{m}$. This second example is a generalization, based on the tariff, of the first example of flexibility pattern.

A third flexibility pattern extends the previous one by introducing the constraint that the cumulated power demand required in an interval $(t, l)$ by the load $i$, i.e., $P_{i}^{D, c u m}$, must be equal to $D_{i}^{\text {cum }}$. In this case, $P_{i}^{D, \text { cum }}=\sum_{h=1, \ldots, m+1} P_{i, h}^{D, \text { cum }}$, where $P_{i, h}^{D, \text { cum }}$ is the cumulated power by the load $i$ in the interval $\left(t_{h}, t_{h}+l_{h}\right)$. Depending on the state of EI and on the tariffs $w_{h}$ associated to each interval, the best configuration (with respect to a cost function) can be considered for $P_{i, 1}^{D, \text { cum }}, \ldots, P_{i, m+1}^{D, \text { cum }}$, such that $P_{i}^{D, \text { cum }}=D_{i}^{\text {cum }}$, if possible. Otherwise, in the worst case the total demand $D_{i}^{\text {cum }}$ has not been completely satisfied at the expiration time $t+l$, a blackout/ cost is incurred. A more general case is obtained, when different (also overlapping) time intervals with different total power demands are considered, each power demand having different level of criticality.

5) Non Flexible Generator: The quantities considered for a non-flexible generator on the bus $i\left(G_{i}\right)$ at time $t$ are: maximum active and reactive power $P_{i}^{\max }$ and $Q_{i}^{\max }$ that a generator can supply; actual active and reactive power $P_{i}^{G}(t)$ and $Q_{i}^{G}(t)$ generated at time $t$ (depending, for renewable generation, from the weather conditions), with $0 \leq P_{i}^{G}(t) \leq P_{i}^{\max }$ and $0 \leq Q_{i}^{G}(t) \leq Q_{i}^{\max }$; voltage $V_{i}(t)$; generation forecast $G_{i}^{F}(t)$ at time $t$ (for renewable generation in controlled area based on weather forecast); tariff (or cost to generate electrical energy) $C_{i}^{G}(t)$. Like for loads, for renewable generation the quantity $G_{i}^{F}(t)$ is the stochastic process representing the generation forecast system. The quantity $P_{i}^{G}(t)-G_{i}^{F}(t)$ represents a measure of generation forecast error for the generator $G_{i}$. Values for the generated power $P_{i}^{G}(t)$ are based on the current power demand at time $t$. Thus, $\stackrel{i}{P}_{i}^{G}(t)$ should be equal to the power $G_{i}^{D}(t)$ required by the loads and supplied by $G_{i}$. For non-flexible generators, $P_{i}^{G}(t)$ cannot be controlled/changed, thus when the power demand $G_{i}^{D}(t)$ changes, then voltage drop can occur on the node $i$; in this case, if the voltage is not maintained within the permissible range, then the generator can trip.

6) Flexible Generator: The parameters associated to a flexible generator on the bus $i\left(F G_{i}\right)$ are the same as those for the non-flexible generators, except that the generated power $P_{i}^{G}(t)$ is flexible. Like for loads, the generated power flexibility can be exploited in: i) time, ii) energy amount or iii) tariff for the user or the cost for the operator. For flexible generators, $P_{i}^{G}(t)$ can be controlled and changed, thus when the power demand $G_{i}^{D}(t)$ changes, then new set points for $P_{i}^{G}(t)$ can be applied. For renewable generators, $P_{i}^{G}(t)$ depends on the weather conditions. Thus, variations of the weather conditions determine new values for $P_{i}^{G}(t)$. When $P_{i}^{G}(t)$ changes, new set points for EI could be required, depending on the difference between the new value of $P_{i}^{G}(t)$ and the old power demand $G_{i}^{D}(t)$ supplied by the generator. In particular, if $P_{i}^{G}(t)>G_{i}^{D}(t)$ then options for the exceeding generated power $P_{i}^{G}(t)-G_{i}^{D}(t)$ could be:

- it is lost (a cost for the operator),

- it is stored into (distributed) storage units (possibly on the same bus of the generator, if any),

- it is supplied to reconfigured flexible loads (such as electrical vehicles, that can accept higher demand),

- it is supplied to non-flexible loads, replacing the (nonrenewable) energy produced by a bulk generator (redispatch of the generated power is required),

- a combination of the above items, depending on the current capacity of the available distributed storage units and on the availability of flexible loads.

For $P_{i}^{G}(t) \leq G_{i}^{D}(t)$ the options for the exceeding power demand $G_{i}^{D} \overline{(t)}-P_{i}^{G}(t)$ could be:

- the power demand $G_{i}^{D}(t)-P_{i}^{G}(t)$ is not met (a cost for the final customer and for the operator),

- it is supplied by (distributed) storage units (possibly on the same bus of the generator, if any),

- it is shifted over time, if it is required by flexible loads,

- it is supplied by another generator (redispatch of the generated power is required),

- a combination of the above items, depending on the current capacity of the available storage units and on the availability of flexible loads. 
7) Distributed Storage Unit: The quantities associated to a distributed storage unit (battery) on the bus $i\left(D S_{i}\right)$ are: storage capacity, i.e., the current amount of electric charge $D S_{i}^{c}(t)$ stored at time $t$ (represents the maximum amount of energy that can be extracted from the battery under certain specified conditions), with $0 \leq D S_{i}^{c} \leq D S_{i}^{c \max }$; maximum capacity $D S_{i}^{c m a x}$, i.e, the maximum amount of electric charge it can store; size $D S_{i}^{\text {size }}$ (the maximum power, typically ranges in 1-10 MW); autonomy $D S_{i}^{\text {auto }}$ (requested in the 10-100 minutes range); efficiency (charge/discharge rate); and expected lifetime (cycles) [14]. Storage units can act as:

- generator: when it can supply energy, if $D S_{i}^{c}(t)>0$,

- load: when it can receive exceeding generated energy, if $D S_{i}^{c}(t)<D S_{i}^{c m a x}$.

They can be associated to distributed generators to reduce the variability of the volatile generation. It is assumed that storage units are operated directly by the Distribution Company in order to increase network control capabilities [14].

8) OLTC: The main quantities considered for an OLTC on the bus $i\left(O L T C_{i}\right)$ are: rated capacity $C_{i}^{O L T C}$, in MVA; primary $V_{i}^{1}$ and secondary $V_{i}^{2}$ voltages; voltage correction per tap $\Delta U_{i}$; number of tap positions $N_{i}^{\text {taps }}$; integer tap position $N_{i}^{\text {tap }}$, with $N_{i}^{\text {mintap }} \leq N_{i}^{\text {tap }} \leq N_{i}^{\text {maxtap }}$; initial tap position $N_{i}^{\text {tapinit }}$, at time 0 ; tap selection time $T_{i}^{\text {tapsel }}$, i.e., the time required to move from one tap position to the next one, that is usually comprised between 3 and 10 seconds; percent impedance $I Z_{i}$, in $\%$; voltage correction factor $C F_{i}^{V}$; active $P_{i}^{O L T C}$ and reactive $Q_{i}^{O L T C}$ power injected on the bus $i$.

9) Capacitor Bank and Voltage Controller: The quantities considered for capacitor bank $\left(C B_{i}\right)$ and voltage control $\left(V C_{i}\right)$ on the bus $i$ are, respectively: reactive power $Q_{i}^{C B}$ injected/ absorbed by $C B_{i}$ on the bus $i$; and voltage $V_{i}^{V C}$ injected or absorbed by $V C_{i}$ on the bus $i$.

\section{FAULT MODEL AND INTERDEPENDENCIES BETWEEN EI AND MCS}

The fault/failure model assumed for EI and MCS is based both on the effects of the faults on the state of EI and both on the accidental and malicious cause of the failures. All faults and failures that may affect a system during its life can be classified according to different basic viewpoints, as shown in [22]. The main failures of (single or multiple) electrical components of the EI could be summarized in:

- Failures involving only the electrical quantities of the components: overloads of power lines, voltage variation outside the regulatory limits (voltage collapse), unexpected reduction of generated power and unexpected increase or reduction of power required by loads.

- Failures involving the topology of the grid $T_{G}$ : disconnection of one or more components, with consequent separation of the components from the electrical network.

A failure involving $T_{G}$ triggers new values for the electrical quantities of EI. The topology $T_{G}$ can change as a results of a failure involving the electrical quantities of EI (e.g., the protections can disconnect a line when the power flow through the line is not maintained below a threshold).
The failures of the MCS components can be summarized in content failures (when the content of the service output deviates from implementing the component function), timing failures (when the timing of output delivery deviates from implementing the component function), halt failure (when the service is halted and the external output becomes constant) and inconsistent failure (when some or all component users perceive differently incorrect service and some users may actually perceive correct service) [22].

Failures in the MCS impact on the state of the EI, i.e. on the electrical quantities and on the topology $T_{G}$, depending on the components affected by the failures, and obviously by the type of the failures. The effects of failures of the MCS components on the overall EI could be:

- wrong application of a reconfiguration, either when effectively required or a spurious one,

- delayed/omitted application of a reconfiguration when necessary (timing or halt failure).

For example, a logical controller $L C$ affected by content failure applies set points with erroneous random values to the controlled component. Failures of the component $L C$ can also impact on the input values (including the information on the state of EI) that the components $L V G C$ (or $M V G C$ ) receive from $L C$. These values can be omitted, delayed (or anticipated) or erroneous. Since, reconfigurations required by $L V G C$ (or $M V G C$ or $C S Y S$ ) are actuated by the associated logical controllers $L C$, a failure of a component $L C$ can also impact on the reconfigurations required by $L V G C$ (or $M V G C$ or $C S Y S$ ). The failure of the components $C S Y S$, $M V G C$ or $L V G C$ corresponds to an erroneous (request of) reconfiguration of the state of the EI (including a non-needed reconfiguration or no reconfiguration) affecting one or more components of the controlled area. The effect of the failure of $C S Y S, M V G C$ or $L V G C$ on a component controlled by $L C$ is the same as the failure of the component $L C$ associated to the controlled component. In the case of inconsistent failure, these effects can be different for each controlled component. The components affected by an inconsistent failure can be selected by a random variable. In general, the failure of the components CSYS, MVGC or $L V G C$ may depend on the failures of the components connected to them by a network. The (content, timing or halt) failure of a communication network $A N$ connecting $L V G C$ and different $L C$ has the same effect of the (content, timing or halt) failure of the $L V G C$. Although, the time to occurrence of the failure and the repair time for $L V G C$ and $A N$ are usually different.

Based on the criteria presented in [22], two classes of faults are defined:

- malicious faults (malicious logic faults and intrusion attempts): human-made faults, introduced with the malicious objective to alter the functioning of the system during use.

- accidental faults: non-malicious faults caused by mistakes or by natural phenomena without human partecipation.

Accidental faults are considered for both the EI and MCS. Whereas, malicious faults are only considered for MCS. Accidental fault can occorring directly in the EI (e.g., a power line broken by a fallen tree), or it can be caused by an accidental or malicious fault occurred in the MCS 
(e.g., erroneous configuration due to an attack of the primary substation controller that generates the trip of a power line). Accidental faults may also directly affect the MCS, producing the failure of one or more entities of the MCS, e.g., the weather forecast system included in the logical component $C S Y S$ or the control communication network associated to an MVGC, corresponding to a logical component $W A N$. Failures of the MCS can be caused by random faults of the EI (as result of a blackout in the area where the MCS components are located), leading to service degradation or failure [23].

Malicious attacks considered in the model are cyber attacks (e.g., flooding based DoS and fake messages) to the control components, e.g., cyber attack to $C S Y S, M V G C, L V G C$, $L C, W A N$ and $A N$, involving the main control functions and their communications. Malicious attacks to MCS can result in delayed/missing data or fake data about measurements, forecasts, set points, etc., thus conveying incorrect informations in the control flow. The impact of cyber attacks on the supplied power depends on: the electrical network size, the amount of distributed generation, the control network topology and the extension of the attack effect [11].

As already shown above, due to the strong interconnection between EI and MCS, a failure in EI propagates to MCS and vice versa (interdependencies), possibly resulting in cascading or escalating failure III. The hybrid-state of EI changes when occurs one of the following events: fault, voltage/var regulation or reconfiguration action by MCS (including erroneous, delayed or not required action) and maintenance actions. MCS actions that change the state of EI can be correctly actived by an event in the EI, or can be erroneously activated by a failure of the MCS. The discrete-state of MCS can change when occurs one of the following events: failure of a component of MCS, recovery from a failure in MCS, fault in EI. Interdependencies from MCS to EI occur, for example, when due to a content failure of MCS a power line could be erroneously open, leading to a disconnection of a part of the electrical network. Interdependencies from EI and MCS occur, for example, when a failure in the EI causes a blackout that reduces the performance of the private or public networks used by MCS, or isolates part of the MCS. Interdependencies from EI and MCS to EI occur, for example, when: a) the MCS fails and does not remove an overload of a power line, the overloaded line open or fails and the topology changes, other lines are overloaded and the disruption propagates; b) the MCS fails and does not control the voltage raising (due to intermittence of renewable units), the voltage upper limit is reached, the generator protection trips, other $D G$ units on the same area will sense the same problem, also their protection trips leading to a sudden voltage drop and the disruption propagates to a set of contiguous $E I$ components, aggravating even more this problem [18].

\section{MEtrics Under EVAluation}

The resilience of a SG system can be evaluated in terms of a variety of measures of interest to final customers, service providers and operators. Especially, blackout-related indicators, as well as performability [24] related ones are among those we consider in our stochastic model based analysis, as listed in the following.

- The percentages $U D(t)$ and $U D(0, t)$ of undelivered power (the undelivered power divided by the power demand) for the whole grid (o for the load $i$ ) at time $t$ or in the interval $[0, t]$, respectively.

- The number of hours of undelivered power demand to load $i, U D_{i}^{H}$, or for the whole grid, $U D^{H}$, from the time of disruption (or time 0 ) until the restoration of the correct state of the power system (that is, after the repair of all the failed components, condition required for all power demand to be met). These measures do not express the loss of power demand in terms of absolute values of power unit, e.g., MW (MegaWatt), but rather in terms of hours of undelivered power demand (until the repair of all the failed components, whose time represents the maximum number of hours of power demand loss that can be experienced). Thus, they provide a intuitive quantification of the loss of power demand that is independent from a reference time window for the analysis, and generalize the measures defined in previous item [25].

- A reward measures $Y(t)$ and $Y(0, t)$ at time $t$ or in the interval $[0, t]$, respectively, based on a reward structure where costs and rewards are considered with respect to the point of the view of the power producers and distributors:

- Costs are associated to power generators, depending on: the quantity of required/produced power, the type of generator, the fault of the generator and the time $t$.

- Rewards are associated to satisfied loads, depending on: the quantity of required/consumed power, the criticality of the load, the time $t$.

- Costs are associated to each interruption of service, depending on: the difference between the required power and the available power for each load, the number of loads which will be powered off, the criticality of loads which will be powered off, duration of the interruption, the time $t$.

- The actual voltage $V_{h}(t)$, active $P_{h}(t)$ and $Q_{h}(t)$ reactive power associated to each electrical component $h$ at time $t$.

- The loss of active power, i.e., the difference between the active power injected by the generators and the active power absorbed by the loads, at time $t, P^{l o s s}(t)$, or in the interval $[0, t], P^{\text {loss }}(0, t)$.

- The number of failed components, at time $t$, or in the interval $[0, t]$.

For flexible generators and loads, the definition of undelivered power (or power demand that is not met) depends on the flexibility pattern considered, as described in Section II-D. The defined measures of interest can be evaluated in terms of mean, variance or distribution.

\section{Modeling And Evaluation Framework}

To model and evaluate the measures of interest introduced in Section IV we first define a stochastic model representing the behavior of the SG system at the needed level of detail, then we define the performance (or reward) variables representing the measures of interest and finally we evaluate the measures by simulation.

\section{A. Important Aspects of the Smart Grid}

The modeling and evaluation framework should be able to represent the following structural and behavioral aspects of the SG systems. 
1) Structural Aspects of the Smart Grid: The SG system has a natural hierarchical structure, as shown in the logical schemes of Figures 3 and 4. At a certain level of detail, the system is composed by many similar components having the same logical structure, as shown, for example, in Figures 1c and 2 for the power lines (arcs) and for the logical components mediumvoltage and low-voltage subtsations (nodes), respectively. Also different instances of the same component $M V G C, L V G C$, $L C$ and $W A N$ and $A N$, as shown in Figure 3, are assumed having the same logical structure. These components can be grouped based on similar sub-components, e.g.: i) all the substations logically structured as a bus and a generator $N^{G}$, ii) or all the substations logically structured as a bus and a load $N^{L}$, iii) or all the substations logically structured as a bus, a generator and a load $N^{G L}$, etc. All similar components can be considered non anonymous replicas (e.g., $N_{i}^{G}, i=1, \ldots, n_{G}$ ) having the same structure and different parameter values for the activities and the events represented. Alternatively, in order to reduce the number of replicated components and to try to improve the efficiency of the resultating model in terms of simulation time, more complex components can be considered for a replica, like as, for example, the triple $N A N$ : substation, power line and substation, i.e. $N A N=\left(N O D E_{1}, A R C, N O D E_{2}\right)$, or, for simplicity, $N A N=N_{1}, A, N_{2}$, where $N_{1}$ and $N_{2}$ represent the starting and ending nodes linked to the power line represented by direct arc $A$. All the logical sub-components defined for $N O D E$ and $A R C$ are included in the model, but disabling (in the final resulting model) all the sub-components of a specific replica that have not to be considered, because they are represented by a different replica. In this example, $n_{A}$ non anonimous replicas of the generic component $N A N$ are considered, one replica $N A N_{l}$ for each arc (or power line) $l$ of the EI network, being $n_{A}$ the number of lines; if, for example, only a generator is associated to the $N O D E_{1}$ of the line $l$, then in the final model, all the other sub-components of the $N O D E_{1}$ are (to be) disabled, except (those representing) the generator and the bus. Following the same approach, also the control components, like as $L C$, can be included in $N A N$, or can be grouped in replicas modeled reparately.

2) Behavioral Aspects of $S G$ : The time to failures of the electrical components depends also on the value of the electrical quantities associated to the components. A failure of a component can propagate to contiguous components. Depending on the failure, the propagation time of a failure could be considered instantaneous. Protections can stop the propagation of a failure by isolating from the grid the component affected by a failure. The activation time of a protection should not be considered instantaneous. The correct activation of a protection depends also on the "strength" of the failure and on the value of the electrical parameters associated to the protection component. The reaction time (with respect to the occurrence of a failure), the failure time and erroneous activation time (when no failure is occurred) of a component (e.g., $M V G C, L V G C$ or $L C$ ) should be considered.

Different functions, with the goal of finding an optimal reconfiguration strategy RS (new set points), should be considered, including voltage/var control algorithms. These functions receive in input the values for $V, \delta_{V}, I, P, Q$ and $T_{G}$, for which EI is not in equilibrium (that is, it is not in acceptable state in terms of costs, voltage, etc.) and outputs the new values for $V, \delta_{V}, I, P, Q$ and $T_{G}$ for which the system EI is in equilibrium (in an acceptable state) and satisfies bounds and constraints (i.e., load balancing, power loss reduction, load shedding, generator redispatch, voltage control, reactive power control, line overload reduction, opening or closing sectioning switches), if possible.

\section{B. Framework's Requirements}

The main features that a modeling and evaluation framework should possess for the analysis of SG, are presented with respect to the following aspects: i) modeling power, i.e., the basic modeling formalisms needed to build the SG model; ii) modeling efficiency, i.e. the advanced modeling mechanisms needed to build the SG model more efficiently; and iii) solution power, i.e., the ability to provide efficient methods to evaluate the measures of interest [26].

1) Modeling power: Representation of continuous, discrete and hybrid states. Time distributions, probability distributions and conditions enabling the time consuming events that can depend both on the discrete and on the continuous state. The call to the functions which describe the effect (in terms of state changes) of the reconfiguration and voltage/var control algorithms. Definition of dependability and performability measures.

2) Modeling efficiency: Hierarchical composition of different sub-models based on replication and composition operators. Replication of anonymous and non anonymous sub-models, sharing part of the state. Compact representation for the topology of the grid (for $T_{G}$ ), for example, describing a part of the state of the system in terms of a incidence matrix [nodes $\mathrm{x}$ arcs]. Compact representation of continuous state for the quantities $V, \delta_{V}, I, P$ and $Q$, describing, for example, a part of the state of the system in term of arrays, associating to each component of EI the corresponding values for $V, \delta_{V}, I, P$ and $Q$ (if any).

3) Solution power: To manage complexity at solution level (like explosion of the states of the model, stiffness and non exponential distributions), ability to perform simulations.

\section{On the Construction of the Overall SG Model with Möbius}

In this Section we address the problem of building the overall modeling framework based on a modular and compositional approach and on the logical schemes proposed in Section II. Although the proposed approach is not related to a specific tool, in order to show how it can be concretely realized, we describe it in terms of a few basic modeling formalisms and mechanisms supported by the tool Möbius, a powerful multiformalism/multisolution tool [27]. The software tool Möbius supports: i) multiple high-level modelling formalisms (SAN, ADVISE, FaultTree, etc.), ii) multiple solution techniques (including simulations), iii) construction of composed models from previously defined models, iv) hierarchical approach to modelling based on state-sharing, v) Join/Rep state-sharing composition node used to compose/replicate submodels, vi) $\mathrm{C}++$ code to define the primitives of the models, as well as custom functions implementing, for example, the required RS. We have selected the SAN and ADVISE formalisms, to model, respectively: i) the stochastic process representing the $\mathrm{SG}$ system, and ii) the attack steps an attacker would execute in order to gain new knowledge or access or to achieve new goals, 
and the adversary profile defining the qualities and interests of an attacker.

The process of constructing the model of a complex system like a SG, based on the manual definition of a lot of submodels, can be very expensive in terms of time and very error prone. The modeling process could be automatized, defining an automated procedure which receives in input the parameters describing the SG (including the topology, the components associated to each node and arc of EI, and the control system MCS), and generates the hierarchical composed model representing the SG. We use an alternative modular and compositional approach, inspired to that proposed in [28] for the electrical transmission networks. It aims to develop a generic and hierarchical composed model based on the composition and replication of template models linked together through sharing of state variables of each model. The overall model represents different smart grid configurations, being input parameters: both the electrical grid topology (represented by a graph) and the components (generators, loads, OLTC, LC, MVGC, LVGC, etc) associated to each node/substation of the grid. With respect to the approach proposed in [28], our approach:

- It copes with the greater complexity due to volatile microgrid generation, flexible generation and loads and newly appliances to control and manage distribution of electricity,

- It simplifies the representation of the dependencies between contiguous components (e.g., nodes and lines of the EI network), thus reducing the complexity of the model and improving the efficiency of the resultating model in terms of simulation time: in [28] the propagation of a fault (e.g., a lightning) through neighboring nodes and lines is explicitely represented by movement of tokens through places of different SAN, triggered by enabling conditions of activities based on the marking of the neighboring components. But here, this type of dependencies are represented, at higher level of abstraction, by single atomic $\mathrm{C}++-$ based reconfiguration actions implemented in the primitives of the SAN, that set a new configurations of the involved components, without triggering a sequence of SAN activities (transitions). Thus, in our approch, the protection mechanism (that, when a fault occurs in an electrical component, triggers the trip of the neighboring components) is not explicitely modeled by the firing of a sequence of SAN activities, but as a result of a single reconfiguration action, that also accounts for the failures of the protection devices.

- It reduces the number of replicated components, by considering more complex logical component for each non anonimous replica. This aims to reduce the simulation time of the model, that, in Möbius, is influenced by the number of replicas and by the complexity of the dependencies of a replica by the state of the other replicas.

The proposed framework is based on templates, i.e., atomic or composed generic model identified as building block. A template represents a group of similar components having: i) the same logical structure, and ii) different parameter values for the activities and the events represented. A specific component of the group represented by a template is defined by non anonymous indexed replicas of the template [28]. Parameters and states of a generic component are defined, respectively, by $(\mathrm{C}++)$ global arrays and array extended places, having one entry for each replica of the template. Each entry of these arrays represents the parameters and the state of a specific component. By applying an index to these arrays, each replica of a template can access to the parameters and the state of the other replicas of the same template, and, if needed, to the parameters and the state of the replicas of other templates.

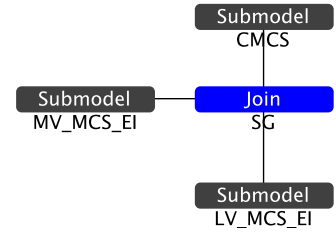

(a)

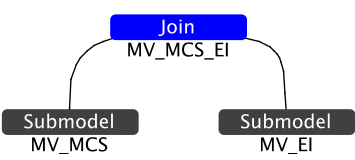

(b)
Fig. 5. Composed models representing the overall SG (a) and MV-MCS and MV-EI (b).

The overall model is obtained by the composition, using the join operator, of the three submodels shown in Figure 5a: CMCS, MV_MCS_EI and LV_MCS_EI; they represent the 3 hierarchical levels of the logical architecture shown in Figure 3.

The model MV_MCS_EI is obtained composing the submodels MV_MCS and MV__EI representing, respectively, MVMCS and MV-EI, as shown in Figure $5 b$.

The construction of the MV_EI model, representing a topology like that shown in Figure $1 \mathrm{~b}$, with $n$ nodes and $m$ arc, comprises the following steps:

1) To define the template models $N 1, N 2$ and $A$, representing the logical components, shown in Figures 2 and 1c, associated to nodes and lines of the network. These



(a)

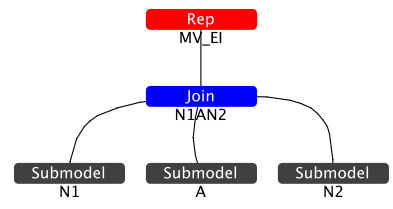

(b)
Fig. 6. Composed models representing the template model for a generic node (a) and for MV-EI (b)

models are obtained by composing the SAN atomic models representing each single component, as shown for $N 1$ in Figure $6 \mathrm{a}$ (for the sake of simplicity, only a subset of the components is considered).

2) To define the hierarchical template model $N 1 A N 2$, representing a generic arc $A$ of the MV-EI network with the associated starting $N 1$ and ending $N 2$ nodes, as described in Section V-A1. The model $N 1 A N 2$ is a hierarchical model generated by composing the submodels $N 1, N 2$, and $A$, as shown in Figure $6 \mathrm{~b}$.

3) To define the hierarchical model MV_EI by automatically replicating $m$ times, one replica for each arc, the template model $N 1 A N 2$, using the rep operator, and assigning at each replica a different index, from 1 to $m$. The index of each replicas is modeled in the template SAN model PL_SAN (representing a generic power line and composing the model $A$ ) through a place AIndex, that is defined 
at time 0 , before enabling the activities representing the behavior of the components. The place AIndex is local to each replica of $N 1 A N 2$, but it is shared between all the atomic SAN composing the model $N 1 A N 2$.

A part of the state of each replica can be represented by the $i$-th entry of an array extended place of $m$ elements/entries (one element for each replica). Each entry can be a $\mathrm{C}++$ plain type (int, real, struct, etc.) or a more complex user defined type. For example, the voltage on the starting node (bus) of the arc (power line) $l$ is represented by the double-type 1-th entry $V 1->\operatorname{Index}(l)->\operatorname{Mark}()$ of the array place $V 1$. The reactive power on the capacitor bank associated to the node $N 1$ of the $\operatorname{arch} l$ is modeled by the double-of-struct-type 1th entry $P 1->\operatorname{Index}(l)->C B->\operatorname{Mark}()$ of the array place $P 1$ (CB is the member of the struct type associated to each entry). These array places are shared between the replicas, thus a replica can access to the state of the other replicas. The parameters and the behavior (marking changes) of each replica can depend on the index of the replica, on the state of the other replicas and on the topology $T_{G}$. The definition of $T_{G}$, for the current configuration of $\mathrm{SG}$, is represented by $\mathrm{C}++$ data structures, statically defined at compilation time. The state of $T_{G}$, representing for example a power line disconnected due to a fault, is represented by array places with $m$ entries, one for each line, like openLines, faultyLines, faultyNode1, etc.

Which sub-models are enabled for each replica, depends on the topology $T_{G}$. For example, if a node $i$ of the network is the starting node of the arcs $l_{1}$ and $l_{2}$, then only one of the two sub-models $N_{1, l_{1}}$ and $N_{1, l_{2}}$ of the replica $N L N_{l}$ is enabled. All the atomic SAN composing a specific replica (i.e, an instance of the model MV_EI) that have not to be considered in the final resulting model (depending of the specific MV-EI network) are permanently disabled, by disabling the enabling conditions of all the activities included in the SAN models; for example, all the enabling conditions in the atomic models representing a component associated to a node $N 1$ connected to the line $l$ are extended by the $\mathrm{C}++$ logical condition $\& \&$ enabled[l].nl, where the parameter enabled[l].nl is defined at compilation time, when the solver is generated for a specific configuration of the modeled SG.

Each SAN atomic model is a template that can represent the main characteristics described in Section II-D for the modeled component. For example, the volatility of a DER due to the weather conditions can be modeled in the SAN DG_SAN like a stochastic process alternating different states of the DER: off (generator is not active), low (generated power is low), medium (generated power is medium) and high (generated power is high); the time spent in each state can be represented by random distributions. Same weather conditions can be modeled for a group of DER located on different nodes, by introducing dependencies between the involved SAN models; dependencies can be defined by synchronizing the activities (transitions) of different SAN on a same shared state. Prediction of power production can be considered in terms of a random error state with respect to the actual production and time spent in each state: none, low, medium or high error. A cost (for example, a higher power loss) can be modeled for each prediction error, because the set points are no more optimal, being based on a weather condition different from the current one. Consequently, also the triggering of new reconfiguration actions for each new erroenous state can be modeled in the SAN model, through state sharing between DG_SAN and MV_MCS model. Similar approaches can be considered to model random power demand flexibility in the atomic model FL_SAN. In particular, random charging demands of arriving electrical vehicles and their assignment to the charging stations, based on the state of EI and the control policies can be represented by a SAN model in term of stochastic process based on a client/server approach (where the loads are servers of random requests of charging).

Using the same approach, it is possible to construct the composed model MV-MCS shown in Figure 7. The atomic



Fig. 7. Composed models representing the template model for MV-MCS.

SAN models LC_SAN, MVGC_SAN and WAN_SAN are the templates representing, respectively, a generic logical controller LC, a generic MVGC and the associated WAN communication network connecting MVGC and LC. The atomic ADVISE models LC_ADVISE and MVGC_ADVISE are the templates representing the adversary profile and the steps to attacks, respectively, LC and MVGC. As shown in Figure 7, these models are composed with the operators join and rep, to obtain $n_{p}$ replicas of the template MVGC_WAN_ATTACK, one replica for each MVGC, and $n_{L C}$ replicas of the template LC_ATTACK, one replica for each LC.

In isolated mode, a replica of LC_SAN models the local control functions/actions performed by an LC to reconfigure the set points of the EI component associated to the LC, depending on the state of the LC. A C++ function controlledComponent (int LCIndex) is statically defined at compilation time to identify the component associated to the replica of LC_SAN with index LCIndex. This function is used to identify the entry of the shared array places of the model MV_EI representing the state (including the values for the set points) of the controlled component, that must be updated.

A replica of MVGC_SAN models the control functions/actions performed by an MVGC to reconfigure the set points of all the EI components controlled by the MVGC, depending on the state of the MVGC. In particular, MVGC_SAN models the condition triggering a control action, the reaction delay (that also depends on the state of the associated WAN), and the evaluation of the reconfiguration strategy (that depends on the states of the controlled EI components, of the associated LC and of the involved communication networks), the attacks to the MVGC and to the WAN and their effetcs. In order to support voltage/var control algorithms, MVGC_SAN model can implement different mixed non-linear optimization problem with bounds and constraints. 


\section{CONCLUSION}

This paper has addressed the stochastic modeling and analysis of Smart Grid systems, to assess indicators useful to quantify the resilience degree of the system. Given the complexity and the size of the tackled problem, the work described here sets the basis towards fulfilling the final objective, that is building a generic, modular and compasable modeling framework for SG evaluation. In particular, the steps accomplished so far include: i) definition of the logical structure of the SG, by identifying its main logical components both at grid and control level; ii) characterization of these components in terms of their state and relationships; iii) definition of a fault model appropriate for the targeted system and a set of relevant resilience-related indicators for the analysis; iv) discussion and identification of the approach to build the modeling framework, with preliminary exemplifications using the SAN formalism. The activities currently in progress as extension of the work presented in this paper go in the direction of: i) implementing the template models as outlined in the last Section, for both the grid components and the attack steps; ii) finding efficient-enough solutions to model the voltage/var control algorithms implemented by the MCS subsystem; iii) select relevant scenarios (e.g., the use cases adopted by the SmartC2Net project) to exercise the modeling framework and practically demonstrate its usefulness and generality.

\section{ACKNOWLEDGMENT}

This work has been partially supported by the European Project SmartC2Net (n. ICT-318023) and the TENACE PRIN Project (n. 20103P34XC) funded by the Italian Ministry of Education, University and Research.

\section{REFERENCES}

[1] D. M. Nicol, W. H. Sanders, and K. S. Trivedi, "Model-based evaluation: From dependability to security," IEEE Trans. Dependable Secure Comput., vol. 1, pp. 48-65, January-March 2004.

[2] S. Chiaradonna, F. Di Giandomenico, and P. Lollini, "Definition, implementation and application of a model-based framework for analyzing interdependencies in electric power systems," Int. J. of Crit. Infrastruct. Prot., vol. 4, pp. 24-40, 2011.

[3] M. Beccuti, S. Chiaradonna, F. Di Giandomenico, S. Donatelli, G. Dondossola, and G. Franceschinis, "Quantification of dependencies between electrical and information infrastructures," Int. J. of Crit. Infrastruct. Prot., vol. 5, pp. 14-27, 2012.

[4] G. D'Agostino, R. Cannata, and V. Rosato, "On modelling of interdependent network infrastructures by extended leontief models," in $4^{\text {th }}$ Int. Workshop on Crit. Inf. Infrastruct. Secur. (CRITIS 2009), ser. LNCS, E. Rome and R. Bloomfield, Eds. Springer Berlin / Heidelberg, 2010, vol. 6027 , pp. 1-13.

[5] R. Bloomfield, L. Buzna, P. Popov, K. Salako, and D. Wright, "Stochastic modelling of the effects of interdependency between critical infrastructures," in $4^{\text {th }}$ Int. Workshop on Crit. Inf. Infrastruct. Secur. (CRITIS 2009), ser. LNCS, E. Rome and R. Bloomfield, Eds. Springer Berlin / Heidelberg, 2010, vol. 6027, pp. 201-212.

[6] D. Kundur, X. Feng, S. Liu, T. Zourntos, and K. Butler-Purry, "Towards a framework for cyber attack impact analysis of the electric smart grid,' in First IEEE Int. Conf. on Smart Grid Commun. (SmartGridComm 2011). IEEE Computer Society, 2010, pp. 244-249.

[7] F. Tabrizi and K. Pattabiraman, "A model for security analysis of smart meters," in $42^{\text {nd }}$ Annu. IEEE/IFIP Int. Conf. on Dependable Syst. and Netw. (DSN 2012). IEEE Computer Society, 2012, pp. 1-6.

[8] The Smart Grid Interoperability Panel - Cyber Security Working Group, "Guidelines for smart grid cyber security: Vol. 3, supportive analyses and references," National Institute of Standards and Technology, NISTIR 7628, August 2010.
[9] S. Muller, H. Georg, C. Rehtanz, and C. Wietfeld, "Hybrid simulation of power systems and ict for real-time applications," in Third IEEE PES Int. Conf. and Exhibit. on Innovative Smart Grid Technol. (ISGT Europe 2012). IEEE Computer Society, 2012, pp. 1-7.

[10] N. Silva, D. Marsh, A. Rodrigues, and C. Mota Pinto, "Dynamic SCADA/DMS data model - plug \& play smart grid solutions," in $21^{\text {st }}$ Int. Conf. and Exhibit. on Electr. Distrib. (CIRED 2011), Frankfurt, June 2011, pp. 1-4, paper N. 1022.

[11] G. Dondossola and R. Terruggia, "SmartC2Net use cases, preliminary architecture and business drivers," FTW, AAU, TUDO, RT, RSE, VO, EFACEC, SmartC2Net WP1 Deliverable: Use Cases and Architecture D1.1, September 2013.

[12] National Grid Education, "How electricity is made and transmitted," http://www.nationalgrideducation.com/ secondary/downloads/education-resources/ngrid_be-the-source_ how-electricity-made-transmitted.pdf, Warwick, UK, 2013.

[13] Working Group Sustainable Processes (SG-CG/SP), "Draft report of the working group sustainable processes to the smart grid coordination group / mandate $\mathrm{m} / 490$ - sg-cg/m490/e_smart grid use case management process - on, management, repository, analysis and harmonization," European Standardization Organizations CEN, CENELEC and ETSI, Tech. Rep., November 2012.

[14] D. Moneta, A. Gelmini, C. Carlini, and M. Belotti, "Storage units: possible improvements for voltage control of MV distribution networks," in $17^{\text {th }}$ Power Syst. Comput. Conf. (PSCC 2011), Stockholm, Sweden, August 2011.

[15] D. Moneta, P. Mora, M. Belotti, and C. Carlini, "Integrating larger RES share in distribution networks: Advanced voltage control and its application on real MV networks," in Integration of Renewables into the Distribution Grid, CIRED Workshop 2012, Lisbon, May 2012, pp. 1-4.

[16] M. Graovac, W. Xiaolin, and R. Iravani, "Integration of storage in electrical distribution systems and its impact on the depth of penetration of DG," Department of Electrical and Computer Engineering, University of Toronto, Prepared for: Chad Abbey, Natural Resources Canada, CANMET Energy Technology Centre, Quebec, Tech. Rep. CETC Number 2009-174 / 2009-10-21, May 2008.

[17] G. Dondossola and R. Terruggia, "USE CASE NAME: Medium voltage control," Ricerca Sul Sistema Energetico - RSE SPA, Italy, SmartC2Net WP1 Deliverable Second Edition 2.0 based on the First Edition 1.0 CEN/CENELE/ETSI SGCG Use Case WGSP-0200, March 2013.

[18] N. Silva, N. Delgado, N. Costa, A. Maia Bernardo, and A. Carrapatoso, "Control architectures to perform voltage regulation on low voltage networks using DG," in Integration of Renewables into the Distribution Grid, CIRED Workshop 2012, Lisbon, May 2012, pp. 1-4, paper N. 351.

[19] X. Feng, W. Peterson, F. Yang, G. M. Wickramasekara, and J. Finney, "Smarter grids are more efficient: voltage and var optimization reduces energy losses and peak demands," ABB Review, no. 3, pp. 33-37, 2009.

[20] C. L. Su, "Comparative analysis of voltage control strategies in distribution networks with distributed generation," in Power \& Energy Society General Meeting, 2009. PES '09. IEEE, Calgary, AB, Canada, July 2009, pp. 1-7.

[21] S. Rahimi, M. Marinelli, and F. Silvestro, "Evaluation of requirements for volt/var control and optimization function in distribution management systems," in $2^{\text {nd }}$ IEEE Int. Energy Conf. and Exhibit. (ENERGYCON 2012), Florence, Italy, September 2012, pp. 331-336.

[22] A. Avizienis, J.-C. Laprie, B. Randell, and C. Landwehr, "Basic concepts and taxonomy of dependable and secure computing," IEEE Trans. Dependable Secure Comput., vol. 1, no. 1, pp. 11-33, 2004.

[23] J. H. Cowie, A. T. Ogielski, B. J. Premore, E. A. Smith, and T. Underwood, "Impact of the 2003 blackouts on internet communications," Renesys Corporation, Preliminary Report, March 2004.

[24] J. F. Meyer, "Performability: A retrospective and some pointers to the future," Performance Evaluation, vol. 4, no. 3-4, pp. 139-156, 1992.

[25] S. Chiaradonna, F. Di Giandomenico, and N. Nostro, "Stochastic assessment of power systems in presence of heterogeneity," Int. J. of Crit. Comp.-Based Syst. - Special Issue on: Dependable and Secure Comput. for Large-Scale Complex Crit. Infrastruct., 2013. 
[26] S. Chiaradonna, F. Di Giandomenico, and P. Lollini, "Case study on critical infrastructures: Assessment of electric power systems," in Resilience Assessment and Evaluation of Computing Systems, K. Wolter, A. Avritzer, M. Vieira, and A. van Moorsel, Eds. Springer Berlin Heidelberg, 2012, pp. 365-390.

[27] T. Courtney, S. Gaonkar, K. Keefe, E. W. D. Rozier, and W. H. Sanders, "Möbius 2.3: An extensible tool for dependability, security, and performance evaluation of large and complex system models," in $39^{\text {th }}$ Annu. IEEE/IFIP Int. Conf. on Dependable Syst. and Netw. (DSN 2009), Estoril, Lisbon, Portugal, June 29-July 2 2009, pp. 353-358.

[28] S. Chiaradonna, P. Lollini, and F. Di Giandomenico, "On a modeling framework for the analysis of interdependencies in electric power systems," in $37^{\text {th }}$ Annu. IEEE/IFIP Int. Conf. on Dependable Syst. and Netw. (DSN 2007), Edinburgh, UK, June 2007, pp. 185-195. 